\title{
Tri-Response Police, Ambulance, Mental Health Crisis Models in Reducing Involuntary Detentions of Mentally Ill People: Protocol for a Systematic Review
}

\author{
Julia Heffernan ${ }^{1, * \mathbb{D}}$, Ewan McDonald ${ }^{1}$, Elizabeth Hughes ${ }^{2}$ and Richard Gray ${ }^{1} \mathbb{C}$ \\ 1 School of Nursing and Midwifery, La Trobe University, Bundoora, Melbourne, VIC 3086, Australia; \\ Ewan.McDonald@latrobe.edu.au (E.M.); R.Gray@latrobe.edu.au (R.G.) \\ 2 Faculty of Medicine and Health, University of Leeds, Leeds LS2 9JT, UK; E.C.Hughes@leeds.ac.uk \\ * Correspondence: J.Heffernan@latrobe.edu.au
}

\section{check for} updates

Citation: Heffernan, J.; McDonald, E.; Hughes, E.; Gray, R. Tri-Response Police, Ambulance, Mental Health Crisis Models in Reducing Involuntary Detentions of Mentally Ill People: Protocol for a Systematic Review. Int. J. Environ. Res. Public Health 2021, 18, 8230. https:// doi.org/10.3390/ijerph18158230

Academic Editor: Gian Maria

Galeazzi

Received: 26 May 2021

Accepted: 28 July 2021

Published: 3 August 2021

Publisher's Note: MDPI stays neutra with regard to jurisdictional claims in published maps and institutional affiliations.

Copyright: (c) 2021 by the authors. Licensee MDPI, Basel, Switzerland. This article is an open access article distributed under the terms and conditions of the Creative Commons Attribution (CC BY) license (https:// creativecommons.org/licenses/by/ $4.0 /)$.

\begin{abstract}
Police, ambulance and mental health tri-response services are a relatively new model of responding to people experiencing mental health crisis in the community, but limited evidence exists examining their efficacy. To date there have been no systematic reviews that have examined the association between the tri-response model and rates of involuntary detentions. A systematic review examining co-response models demonstrated possible reduction in involuntary detention, however, recommended further research. The aim of this protocol is to describe how we will systematically review the evidence base around the relationship of the police, ambulance mental health tri-response models in reducing involuntary detentions. We will search health, policing and grey literature databases and include clinical evaluations of any design. Risk of bias will be determined using the Effective Public Health Practice Project Quality Assessment Tool and a narrative synthesis will be undertaken to synthesis key themes. Risk of bias and extracted data will be summarized in tables and results synthesis tabulated to identify patterns within the included studies. The findings will inform future research into the effectiveness of tri-response police, ambulance, and mental health models in reducing involuntary detentions.
\end{abstract}

Keywords: mental health; mental illness; police; ambulance; pacer; psychosis; self-harm; involuntary detention; section; assessment; systematic review; protocol

\section{Introduction}

Involuntary detention is a common mechanism used to compel people who appear acutely mentally ill for a mandatory psychiatric assessment or period of observation [1-5]. It is a controversial power provided in mental health legislation, generally to doctors, mental health workers, police officers and in some areas, paramedics. Involuntarily detaining a person brings with it additional powers which allow force to be used upon the person to complete that detention as included in mental health legislation [5,6]. Such powers may include forcing entry into the persons property, searching their person and property, using physical force and restraint, and the use of chemical sedation [1-5].

Mental health consumers and carers report that involuntary detention is a traumatic, humiliating and often frightening experience, particularly when involving police or law enforcement agencies, which negatively impacts their overall mental wellbeing [1-11]. It is consistently demonstrated that involuntary detention invokes loss of perceived independence, worsening of paranoid beliefs, terror and distress, re-traumatization, and powerlessness, particularly for those who experienced restrictive practices such as restraint and forcible giving of medications [1-11].

Police will often invoke involuntary detention in the absence of other mechanisms to ensure prompt assessment of a person experiencing mental illness [2,12,13]. Similarly, ambulance paramedics frequently respond to mental health crisis, often co-responding with 
police $[14,15]$. High rates of involuntary detentions have significant resourcing impacts on emergency services and particularly emergency departments (EDs) and hospitals who are required to undertake the assessments. This includes higher numbers of patients in EDs, the need for greater supervision, prevention of absconding, and pressures related to involuntary detention assessment times [16-19].

Research suggests significant increases in the rates of involuntary detentions [13,16-20]. An analysis of involuntary detention rates in the United States determined that the rate of involuntary detentions in 22 states was increasing by 13\% every year between 2012 to 2016 [17]. As a result, health services, police and ambulance services have observed the need to provide mental health expertise directly into police and ambulance presentations involving mental health patients.

A trained mental health clinician can provide expert assessment in the field, negating the need to invoke involuntary detention or transporting the person to hospital for assessment [3,21]. A number of models are being trialled across a number of developed countries including adding mental health workers into police or ambulance call centers, coresponse mobile crisis services which may team a mental health worker with a paramedic or police officer, or the tri-response model which incorporates all three agencies [21]. Police, Ambulance, Clinician Early Response (PACER) is a tri-response mobile service which teams a mental health clinician, police officer and ambulance paramedic together in a first responder vehicle to attend mental health crisis in the community and is one of a number of models being trialled to meet this need, yet it requires further exploration to assess its efficacy in reducing involuntary detentions. Currently, tri-response models are operating in parts of Australia, the United Kingdom, the United States and Europe.

A systematic review of police mental health co-responder models was undertaken in 2018 to identify and describe the different models, identify the types of service users who came in contact with the models and to evaluate their effectiveness [21]. The authors included 26 papers into the review and concluded that the co-responder police mental health models may reduce rates of involuntary detention of mentally ill people [21]. However, they opined that further research was required, and at the time of writing, no review has been conducted to evaluate the tri-service model [21].

\section{Research Objectives}

This protocol describes the objective of the future review which is to synthesize the available evidence regarding the effects of the PACER mobile crisis service and similar triresponse models in diverting patients from hospital and reducing unnecessary involuntary detention.

1. To clarify the evidence base around the relationship of the tri-response model in reducing involuntary detentions of people experiencing mental health crisis. This systematic review will clarify the available research through all available studies published in journals and abstracts, which meet inclusion criteria.

2. To compare the rate of tri-response involuntary detentions which convert to hospitalizations with involuntary detentions made by police and/or ambulance paramedics.

3. To compare the rate of involuntary detentions by the tri-response model with rates of involuntary detentions made by police and/or ambulance paramedics.

\section{Materials and Methods}

\subsection{Review Question}

The aim of the systematic review is to determine the safety and effectiveness of the tri-response crisis model compared with routine intervention in people experiencing a psychiatric crisis. This protocol sets out the following review design. 


\subsection{Design}

We will include clinical studies of any design which answers the research question and exclude qualitative studies. Our protocol complied with the Preferred Reporting Items for Systematic Reviews and Meta-Analyses Protocols checklist 2015 (PRISMA-P) [11].

Our review is registered with OSF: doe:10.17605/OSF.IO/3EMRV.

\subsection{Eligibility Criteria}

Participants are experiencing an acute mental health crisis which has precipitated an emergency response or PACER response. Acute as defined for the purpose of this systematic review, pertains to a mental health presentation which is severe or intense in nature, and poses a risk to the person or others because of mental illness. It refers to the need for immediate assessment for which an emergency response is appropriate. Examples of such presentations include florid psychosis, mania, suicidal behaviour, and behavioural disturbance. Presentations can be unknown to the responders and requiring crisis assessment. Such presentations may include a person behaving in a confused and agitated manner in a public place.

We will include studies meeting the following inclusion criteria:

- $\quad$ Patients meeting the participant criteria;

- The exposure or intervention is tri-response police, ambulance, mental health models;

- All participant demographics (age, gender, ethnicity, etc.);

- All countries of publication;

- Published in English.

\subsection{Exclusion Criteria}

The focus of the review described in this protocol is to synthesis the evidence relating to the tri-response PACER model; therefore, co-response models will be excluded. Coresponse models include police and mental health clinician; paramedic and mental health clinician; non-mobile models, mental health clinicians embedded within police station or emergency communications centers. For example, some known services are titled "PACER" but stand for "Police and Clinician Early Response" and do not include an ambulance paramedic. Others are termed "psychiatric ambulances" and refer to a model where a clinician is embedded with a paramedic team in an ambulance vehicle. It is considered that the co-response model is not representative of the tri-response model.

\subsection{Searching the Grey Literature}

Grey literature will be used in the review and is expected to include service evaluations of tri-response PACER models, relevant theses or dissertations, research and committee reports, and government reports. Reference lists of relevant studies, citation searching and searching relevant internet resources will also be included

\subsection{Comparator Intentions}

Any comparator will be included in the review however the primary comparator is a standard emergency service response, that refers to a patient experiencing mental health crisis who is reviewed by an ambulance or police team, as part of standard emergency service response.

\subsection{Search Strategy}

The Medline search strategy can be found in Table 1. This review will incorporate both automated and manual searches. Literature relating to tri response PACER models will most likely come from health databases, however police databases will also be searched including:

Health databases:

- CINAHL Complete; 
- Health and Medical Complete (ProQuest);

- $\quad$ Medline (OVID);

- Joanna Briggs Institute EBP Database;

- Health Collection (Informit);

- PsychINFO;

- PsycARTICLES;

- ANZCTR.

Policing databases:

- ProQuest Criminal Justice Database;

- Australian Federal Police Digest;

- $\mathrm{CINCH}$ Australian Criminology Database.

Gray literature databases:

- Open Gray;

- Gray Source;

- ProQuest:

- Google.

Table 1. Medline Search.

\begin{tabular}{|c|c|c|}
\hline Search ID & Search Term & PICO Element \\
\hline S1 & Mental Health & Population \\
\hline S2 & Psychiatr* & \\
\hline S3 & Mental* Ill* $^{*}$ & \\
\hline S4 & Crisis & \\
\hline S5 & Emergency & \\
\hline S6 & Acute* & \\
\hline S7 & Urgent & \\
\hline S8 & Relapse & \\
\hline S9 & Self-harm* & \\
\hline S10 & Self-injur* & \\
\hline S11 & Psycho* & \\
\hline S12 & Mani* & \\
\hline S13 & Suicid ${ }^{*}$ & \\
\hline S14 & $\mathrm{S} 1$ or $\mathrm{S} 2$ or $\mathrm{S} 3$ and $\mathrm{S} 4$ or $\mathrm{S} 5$ or $\mathrm{S} 6$ or $\mathrm{S} 7$ or $\mathrm{S} 8$ & \\
\hline S15 & $\mathrm{S} 14$ and $\mathrm{S} 9$ or S10 or S11 or S12 or S13 & \\
\hline S16 & PACER $*$ & Intervention \\
\hline S17 & Police & \\
\hline S18 & Ambulance & \\
\hline S19 & Nurse & \\
\hline S20 & Paramedic & \\
\hline S21 & Law Enforcement & \\
\hline S22 & Street Triage & \\
\hline S23 & Joint & \\
\hline S24 & Mobile & \\
\hline S25 & Tri-response & \\
\hline S26 & $\mathrm{S} 16$ or S17 or S18 or S19 or S20 or S21 & \\
\hline S27 & $\mathrm{S} 22$ or S23 or 24 or S25 & \\
\hline S28 & Emergency Service* or S26 & Comparator \\
\hline S29 & Hospital ${ }^{*}$ & Outcome \\
\hline S30 & Section* & \\
\hline S31 & Involuntary & \\
\hline S32 & Detention & \\
\hline S33 & Section 136 & \\
\hline S34 & Legislation & \\
\hline S34 & $\mathrm{S} 29$ or S30 or S31 or S32 or s33 OR s34 & \\
\hline
\end{tabular}

To maximize outcomes of the literature search, we will ensure that synonyms are utilised as part of the search strategy. Several synonyms exist in this field of study and 
relate to the description of mental health patients, terminology used to describe involuntary detentions and the title of PACER. For example, "mental health" is a field of psychiatry and the term is interchangeable with "mental illness". Involuntary detentions are commonly referred to as "sections" with specific countries and states referencing the individual section within their own legislation. Therefore, Boolean operators, plurals, truncations, and wildcards will be included in the search terms to maximize the search results and reduce researcher burden.

Furthermore, "PACER" is a commonly used term in the medical field of cardiology and will need to be paired with a cardiac search term exclusion. It is likely that the search will produce many co-response model studies despite strict search terms and will then need to undergo further screening to identify those which refer to the PACER tri-response. Finally, the search will need to filter out the clinician, given this is a highly changeable term which is unlikely to appear in many titles or abstracts and may impact the outcome of the search.

\subsection{Data Retrieval}

The selected studies will be exported from bibliographic databases to reference management software (Endnote)

\subsection{Data Screening}

Duplicate references will be removed in Endnote and citations exported into Covidence, a web-based software platform for systematic reviews including citation screening, review of full text articles, risk of bias assessment, extraction of study characteristics and outcomes, and exportation of data.

We will use two reviewers to review citations for inclusion in the review, initially using title and abstracts to screen against inclusion/exclusion criteria. Using this double screening approach which offers the following advantages: assurance that the inclusion/exclusion criteria are consistently applied, identification of and correction of mistakes, and avoidance of systematic errors [22].

Conflict will be resolved through discussion and any issues that are unable to be resolved will be referred to a third reviewer for resolution. This screening process aims to avoid inclusion of evidence with a subsequent risk of bias that could endanger the validity of the conclusions drawn in the review. These requirements aim to avoid the nondetection of relevant evidence with a subsequent risk of bias that endangers the validity of conclusions drawn from the evidence available. The relevant publications are selected in several steps. Following the first screening phase, full text screening will occur using the same process of double screening against the inclusion/exclusion criteria.

\subsection{Data Extraction}

Data will be extracted from papers using an extraction tool developed for this review using the review question as a guide. The data extracted will include details about the intervention, patient population, study methods, and outcomes of significance to the review question and objectives.

\subsection{Risk of Bias in Individual Studies}

Quality assessment of the included literature will be carried out at the point of data extraction. The Effective Public Health Practice Project Quality Assessment Tool (EPHPP) (https://merst.ca/wp-content/uploads /2018/02/quality-assessment-tool_2010.pdf, accessed on 18 April 2021). The EPHPP was developed in Canada by the Effective Public Health Practice Project and is an effective tool for evaluating a number of different study designs including Randomised Controlled Trials (RCTs), before and after intervention studies and case-control studies [23].

The tool has been assessed as having content and construct validity and measures six key domains: (1) selection bias; (2) study design; (3) confounders; (4) blinding; (5) method 
of data collection; and (6) withdrawals and dropouts, and two further components of intervention integrity and analyses [13]. The EPHPP was assessed for content validity using an iterative process of systematic repetition of sequences of testing different data [24]. The tool was reviewed for consistency of coding, interpretation, and examination of evidence tables using an expert group. The authors further assessed the validation process through evaluating the content of the tool and the individual categories for clarity, completeness, and relevance, as well and comparing the EPHPP with similar tools [24]. Furthermore, Test-retest reliability of the EPHPP was calculated over two occasions using two reviewers and a random selection of studies, with agreement between the two reviewers (Kappa 0.74: Kappa 0:61) [24].

Overall, the EPHPP has a strong methodological rating based upon its validity and reliability [24]. For this review, two reviewers will use the EPHPP to rate the study quality and a comparison of individual ratings will reach a consensus on each component. In the event of a lack of consensus, a third reviewer will apply the EPHPP to the contested study. The overall study quality will be rated based upon the combined component ratings using the following:

Strong-Four strong ratings with no weak ratings;

Moderate-Less than four strong rating and one weak rating;

Weak-Two or more weak ratings.

\subsection{Data Synthesis}

Narrative synthesis will be performed due to the inclusion of all study designs in the review which may yield literature that is not adequately clinically homogenous to allow for meta-analysis [25-28].

The synthesis will focus upon the intervention implementation and effect and grouped into themes. The synthesis will be undertaken and carried out using the following framework:

- Developing a theory of how the intervention works, why and for whom;

- Developing a preliminary synthesis of findings and included studies;

- Exploring relationships within and between studies;

- Assessing the robustness of the synthesis [29].

A preliminary synthesis of the findings of the studies and grey literature will be completed using the Cochrane Narrative Synthesis Advice [30]. Results will be tabulated to identify the patterns within the studies including population size, length of study, study design and outcomes and then transcribed into both descriptive and statistical format.

\subsection{Meta Bias(es)}

Bias refers to systematic error that skews the results of a study in a particular direction and leads to the acceptance of outcomes or results without considering the likelihood of unfair or misleading presentation [31-33]. There are many types of bias that can impact the findings of a systematic review. Publication bias refers to the inclusion of studies which are statistically significant or demonstrate favourable results but minimize the inclusion of studies which do not [31-33]. Studies which demonstrate efficacy, success, or confirm a researcher's hypothesis, are more likely to be published and can threaten the validity of the review, providing an unbalanced summary of the evidence [33]. Publication bias can be minimized by the inclusion of grey literature in the review [31-33]. Removing grey literature from meta-analysis results in $15 \%$ larger estimates of treatment effects, less precise effect-size estimates and more significant results and using only published trials may result in greater treatment effect [33] (p. 234).

Similarly, selective reporting or selection bias occurs when research results are deliberately reported inaccurately in order to suppress or exclude negative or undesirable findings [31,32] This may result in findings which are skewed and unable to be reproduced in further study. The impact of selection bias can vary and may be influenced by the selector's knowledge of the subject, existing collaborations, and informed opinion about 
the topic [31,32]. Where available, we will assess study protocols of literature to be included in the review and determine the completeness of the reporting. Selection bias can further be reduced by the inclusion of grey literature [33].

Inclusion of grey literature alone does not entirely alleviate the risk of publication bias or selective reporting. To minimize systematic error of the above biases, we will use the funnel plot measure of symmetry of the study effect in the included literature [33].

\section{Results}

Risk of bias and extracted data will be summarized and emphasized through tables, graphs and other diagrams used to compare the data [26]. Where possible point estimates (the value that represents a best estimate of effects) and interval estimates (an estimated range of effect, presented as a 95\% confidence interval) will be presented in the results [26].

\section{Discussion}

This paper sets out the protocol for a planned systematic review and narrative synthesis. Each step has been carefully considered and planned to meet best practice standards for systematic reviews of health research.

\section{Conclusions}

There is considerable interest from consumer and carer groups to reduce the use of involuntary detention and restrictive practices of people experiencing mental illness, and to promote recovery and least restrictive care. This review will add to the body of evidence about tri-response mobile crisis response teams in responding to people in mental health crisis, and their capacity to reduce involuntary detentions.

\section{Relevance to Clinical Practice}

There is considerable interest from consumer and carer groups to reduce the use of involuntary detention and restrictive practices of people experiencing mental illness, and to promote recovery and least restrictive care. This review will add to the body of evidence about cross-agency mobile crisis response teams in responding to people in mental health crisis, and their capacity to reduce involuntary detentions.

Author Contributions: J.H. conceptualized, prepared, and wrote the review protocol and original draft. R.G., E.M. and E.H. supervised the review and edited the manuscript. All authors independently reviewed the search strategy. All authors have read and agreed to the published version of the manuscript.

Funding: This research received no external funding.

Institutional Review Board Statement: Not applicable.

Informed Consent Statement: Not applicable.

Acknowledgments: The authors acknowledge the Canberra Hospital Library Consultancy Service, ACT Health, Canberra, Australian Capital Territory, Australia for prospectively undertaking the literature search.

Conflicts of Interest: The authors declare no conflict of interest.

\section{References}

1. Akther, S.F.; Molyneaux, E.; Stuart, R.; Johnson, S.; Simpson, A.; Oram, S. Patients' experiences of assessment and detention under mental health legislation: Systematic review and qualitative meta-synthesis. BJPsych Open 2019, 5, e37. [CrossRef]

2. Feeney, A.; Umama-Agada, E.; Curley, A.; Asghar, M.; Kelly, B.D. Police Involvement in Involuntary Psychiatry Admission: A Report from the Dublin Involuntary Admission Study. Psychiatr. Serv. 2020, 71, 1292-1295. [CrossRef]

3. Keown, P.; French, J.; Gibson, G.; Newton, E.; Cull, S.; Brown, P.; Parry, J.; Lyons, D.; McKinnon, I. Too much detention? Street Triage and detentions under Section 136 Mental Health Act in the North-East of England: A descriptive study of the effects of a Street Triage intervention. BMJ Open 2016, 6, e011837. [CrossRef] 
4. $\quad$ Rains, L.S.; Zenina, T.; Dias, M.C.; Jones, R.; Jeffreys, S.; Branthonne-Foster, S.; Lloyd-Evans, B.; Johnson, S. Variations in patterns of involuntary hospitalisation and in legal frameworks: An international comparative study. Lancet Psychiatry 2019, 6, $403-417$. [CrossRef]

5. Saya, A.; Brugnoli, C.; Piazzi, G.; Liberato, D.; Di Ciaccia, G.; Niolu, C.; Siracusano, A. Criteria, Procedures, and Future Prospects of Involuntary Treatment in Psychiatry Around the World: A Narrative Review. Front. Psychiatry 2019, 10, 271. [CrossRef]

6. Seed, T.; Fox, J.R.; Berry, K. The Experience of Involuntary Detention in Acute Psychiatric Care. A Review and Synthesis of Qualitative Studies. Int. J. Nurs. Stud. 2016, 61, 82-94. [CrossRef] [PubMed]

7. Bainbridge, E.; Hallahan, B.; McGuinness, D.; Gunning, P.; Newell, J.; Higgins, A.; Murphy, K.; McDonald, C. Predictors of involuntary patients' satisfaction with care: Prospective study. BJPsych Open 2018, 4, 492-500. [CrossRef]

8. Bradbury, J.; Hutchinson, M.; Stasa, H.; Hurley, J. Lived experience of involuntary transport under mental health legislation. Int. J. Ment. Health Nurs. 2016, 26, 580-592. [CrossRef]

9. Murphy, R.; McGuinness, D.; Bainbridge, E.; Brosnan, L.; Felzmann, H.; Keys, M.; Murphy, K.; Hallahan, B.; McDonald, C.; Higgins, A. Service Users? Experiences of Involuntary Hospital Admission Under the Mental Health Act 2001 in the Republic of Ireland. Psychiatr. Serv. 2017, 68, 1127-1135. [CrossRef]

10. Riley, G.; Freeman, E.; Laidlaw, J.; Pugh, D. “A frightening experience": Detainee's and carers experience of being detained under section 136 of the mental health act. Med. Sci. Law 2011, 51, 164-169. [CrossRef]

11. Stuart, R.; Akther, S.F.; Machin, K.; Persaud, K.; Simpson, A.; Johnson, S.; Oram, S. Carers' experiences of involuntary admission under mental health legislation: Systematic review and qualitative meta-synthesis. BJPsych Open 2020, 6, e19. [CrossRef]

12. Godfredson, J.W.; Thomas, S.D.; Ogloff, J.R.; Luebbers, S. Police perceptions of their encounters with individuals experiencing mental illness: A Victorian survey. Aust. N. Z. J. Criminol. 2011, 44, 180-195. [CrossRef]

13. Thomas, A.; Forrester-Jones, R. Understanding the Changing Patterns of Behaviour Leading to Increased Detentions by the Police under Section 136 of the Mental Health Act 1983. Polic. A J. Policy Pract. 2018, 13, 134-146. [CrossRef]

14. Duncan, E.A.S.; Best, C.; Dougall, N.; Skar, S.; Evans, J.; Corfield, A.R.; Fitzpatrick, D.; Goldie, I.; Maxwell, M.; Snooks, H.; et al. Epidemiology of emergency ambulance service calls related to mental health problems and self harm: A national record linkage study. Scand. J. Trauma Resusc. Emerg. Med. 2019, 27, 34. [CrossRef]

15. Rees, N.; Rapport, F.; Snooks, H. Perceptions of paramedics and emergency staff about the care they provide to people who self-harm: Constructivist metasynthesis of the qualitative literature. J. Psychosom. Res. 2015, 78, 529-535. [CrossRef]

16. Australian Institute of Health and Welfare. Emergency Department Care 2017-2018: Australian Hospital Statistics; Health Services Series no 89; AIHW: Canberra, Australia, 2018. Available online: https:/ /www.aihw.gov.au/getmedia/9ca4c770-3c3b-42fe-b071 -3d758711c23a/aihw-hse-216.pdf.aspx?inline=true (accessed on 13 May 2021).

17. Lee, G.; Cohen, D. Incidences of Involuntary Psychiatric Detentions in 25 U.S. States. Psychiatr. Serv. 2021, 72, 61-68. [CrossRef] [PubMed]

18. New York City Department of Health and Mental Hygiene. Epi Data Brief-Mental Health Emergency Department Visits among New York City Adults, 2015. 2018. Available online: https://www1.nyc.gov/assets/doh/downloads/pdf/epi/databrief107.pdf (accessed on 13 May 2021).

19. NHS Digital. Hospital Accident and Emergency Activity, 2017-2018. 2019. Available online: https://digital.nhs.uk/data-andinformation/publications/statistical/hospital-accident--emergency-activity/2017-18 (accessed on 13 May 2021).

20. Smith, S.; Gate, R.; Ariyo, K.; Saunders, R.; Taylor, C.; Bhui, K.; Mavranezouli, I.; Heslin, M.; Greenwood, H.; Matthews, H.; et al. Reasons behind the rising rate of involuntary admissions under the Mental Health Act (1983): Service use and cost impact. Int. J. Law Psychiatry 2020, 68, 101506. [CrossRef]

21. Puntis, S.; Perfect, D.; Kirubarajan, A.; Bolton, S.; Davies, F.; Hayes, A.; Harriss, E.; Molodynski, A. A systematic review of co-responder models of police mental health 'street' triage. BMC Psychiatry 2018, 18, 1-11. [CrossRef] [PubMed]

22. Waffenschmidt, S.; Knelangen, M.; Sieben, W.; Bühn, S.; Pieper, D. Single screening versus conventional double screening for study selection in systematic reviews: A methodological systematic review. BMC Med. Res. Methodol. 2019, 19, 1-9. [CrossRef]

23. Armijo-Olivo, S.; Stiles, C.R.; Hagen, N.A.; Biondo, P.D.; Cummings, G.G. Assessment of study quality for systematic reviews: A comparison of the Cochrane Collaboration Risk of Bias Tool and the Effective Public Health Practice Project Quality Assessment Tool: Methodological research. J. Eval. Clin. Pract. 2010, 18, 12-18. [CrossRef]

24. Thomas, B.; Ciliska, D.; Dobbins, M.; Micucci, S. A Process for Systematically Reviewing the Literature: Providing the Research Evidence for Public Health Nursing Interventions. Worldviews Evid. Based Nurs. 2004, 1, 176-184. [CrossRef]

25. Deeks, J.; Higgins, J.; Altman, D. Chapter 10: Analysing data and undertaking meta-analysis. In Cochrane Handbook for Systematic Reviews of Interventions Version 6.0; Higgins, J., Thomas, J., Chandler, J., Cumpston, M., Li, T., Page, M., Walsh, V., Eds.; Cochrane: London, UK, 2019; Available online: www.training.cochrane.org/handbook (accessed on 11 February 2021).

26. Lockwood, C.; White, S. Synthesizing Descriptive Evidence; Lippincott Williams \& Wilkins/Joanna Briggs Institute: Adelaide, SA, Australia, 2012.

27. McKenzie, J.; Brennan, S.; Ryan, R.; Thompson, H.; Johnston, R.; Thomas, J. Chapter 3: Defining the criteria for including studies and how they will be grouped for synthesis. In Cochrane Handbook for Systematic Reviews of Interventions Version 6.0; Higgins, J., Thomas, J., Chandler, J., Cumpston, M., Li, T., Page, M., Walsh, V., Eds.; Cochrane: London, UK, 2019. Available online: https: / / training.cochrane.org/handbook/current/chapter-03 (accessed on 21 December 2020). 
28. Ryan, R.; Cochrane Consumers and Communication Review Group. 'Heterogeneity and Subgroup Analysis in Cochrane Consumers and Communication Group Reviews: Planning the Analysis at Protocol Stage. 2016. Available online: http: / / cccrg.cochrane.org (accessed on 21 December 2020).

29. Leamy, M.; Bird, V.; Le Boutillier, C.; Williams, J.; Slade, M. Conceptual framework for personal recovery in mental health: Systematic review and narrative synthesis. Br. J. Psychiatry 2011, 199, 445-452. [CrossRef]

30. Moher, D.; Liberati, A.; Tetzlaff, J.; Altman, D.G.; The PRISMA Group. Preferred Reporting Items for Systematic Reviews and Meta-Analyses: The PRISMA Statement. PLoS Med. 2009, 6, e1000097. [CrossRef]

31. Ahmed, I.; Sutton, A.J.; Riley, R.D. Assessment of publication bias, selection bias, and unavailable data in meta-analyses using individual participant data: A database survey. BMJ 2012, 344, d7762. [CrossRef] [PubMed]

32. Ayorinde, A.A.; Williams, I.; Mannion, R.; Song, F.; Skrybant, M.; Lilford, R.J.; Chen, Y.-F. Assessment of publication bias and outcome reporting bias in systematic reviews of health services and delivery research: A meta-epidemiological study. PLoS ONE 2020, 15, e0227580. [CrossRef] [PubMed]

33. Paez, A. Gray literature: An important resource in systematic reviews. J. Evid. Based Med. 2017, 10, 233-240. [CrossRef] [PubMed] 